\title{
Metabolic Imaging of Ischemic Stroke: The Present and Future
}

\author{
K.A. Dani and S. Warach
}

\begin{abstract}
SUMMARY: Measures of cerebral metabolism may be useful in the selection of patients for reperfusion therapies and as end points in clinical trials. However, there are currently no clinically routine techniques that provide such data directly. We review how imaging modalities in current clinical use may provide surrogate markers of metabolic activity. Promising techniques for metabolic imaging that are currently in the pipeline are reviewed.
\end{abstract}

ABBREVIATIONS: $\mathrm{MR}-\mathrm{COMI}=$ cerebral oxygen metabolic index; $\mathrm{CMRO}_{2}=$ cerebral metabolic rate for oxygen; $\mathrm{OCl}=$ oxygen challenge imaging; $\mathrm{OEF}=$ oxygen extraction fraction

O ver the past 2 decades, imaging markers that predict outcome after reperfusion therapies for acute ischemic stroke have continually been refined. Despite the promise offered by PWI and DWI MR studies to advance patient selection for reperfusion therapies beyond noncontrast CT, there remains a thirst to develop further techniques that may potentially provide more information about the cerebral pathophysiology of an individual patient. This may be achieved by targeting any one of the plethora of pathophysiologic consequences of stroke, the end point of which is reduced or absent metabolic activity. We review how measures of metabolic activity may be inferred from current and promising techniques (Fig 1).

\section{Why Image Metabolism?}

After arterial occlusion, CBF diminishes precipitously. However, tissue does not die immediately but enters a twilight state of electrical silence associated with maintained cerebral metabolic rate for oxygen $\left(\mathrm{CMRO}_{2}\right)$ - the ischemic penumbra. ${ }^{1}$ The first adaptation to hypoperfusion is rapid dilation of the precapillary arterioles, which increases CBV. The next is an increase in the proportion of oxygen extracted from the blood (oxygen extraction fraction, OEF), which occurs concomitantly with a switch from

\footnotetext{
Received August 8, 2013; accepted after revision August 27.

From the Institute of Neurosciences and Psychology (K.A.D.), University of Glasgow, Institute of Neurological Sciences, Glasgow, United Kingdom; and Department of Neurology and Neurotherapeutics (S.W.), UT Southwestern, Dallas, Texas. Please address correspondence to Steven Warach, Department of Neurology and Neurotherapeutics, UT Southwestern, 5323 Harry Hines Blvd, Dallas, TX 75390-8813; e-mail: swarach@seton.org

- Indicates open access to non-subscribers at www.ajnr.org

http://dx.doi.org/10.3174/ajnr.A3789
}

aerobic to anaerobic metabolism. At the same time, however, a cascade of pathologic processes is set in motion including excitotoxicity, peri-infarct depolarization, oxidative stress, inflammation, and apoptosis. It is now appreciated that if there is reperfusion, tissue may be salvaged and infarction averted, and this is associated with clinical improvement. ${ }^{2}$ The NINDS trial ${ }^{3}$ was the first to show that reperfusion therapy with rtPA is beneficial when administered to patients with ischemic stroke presenting within a restricted time window, after NCCT has excluded major established infarction. However, there are a number of acknowledged limitations, including the small but significant risk of intracerebral hemorrhage after alteplase administration, the observation that not all patients benefit, and the increasing recognition that some patients beyond the current time window also have penumbral tissue. ${ }^{4}$ A goal of recent imaging developments has been to refine patient selection by not only identifying those patients with volumes of infarct core likely to predispose to hemorrhagic transformation but also by identifying those patients with large volumes of ischemic penumbra. The advanced imaging techniques that are the subject of most attention measure perfusion parameters (PWI) and cytotoxic edema (DWI). However, given that penumbra and core are defined by metabolic parameters, the ability to image metabolic activity directly may further aid patient selection for therapies. Ultimately, such imaging may be complementary to the use of the "time window" and PWIDWI data. Metabolic imaging may also potentially guide future treatments in the subacute and chronic time periods and facilitate clinical trial end points.

\section{PET: The Reference Standard}

Although the use of PET in stroke remains a research tool, this technique deserves special mention because PET data have clarified im- 
Pathophysiologica

Process
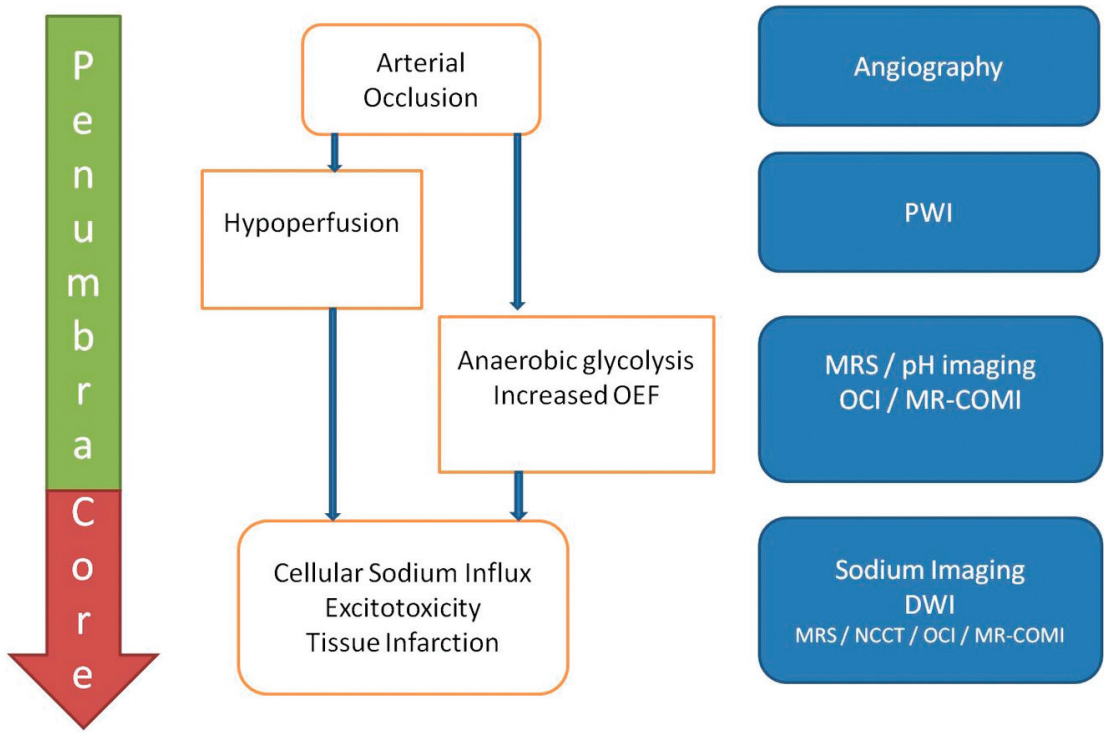

Sodium Imaging

DWI

MRS / NCCT / OCI / MR-COM

FIG 1. Approach to metabolic imaging of acute stroke. On the left, the tissue compartments (penumbra/core) are delineated. In the middle, the pathophysiologic process is outlined. On the right, and aligned in the horizontal meridian, imaging techniques that may image the respective pathophysiologic process are documented.

portant principles regarding stroke pathophysiology. ${ }^{15} \mathrm{O}$ multitracer PET can provide measures of not only CBV and CBF but also $\mathrm{OEF}$ and $\mathrm{CMRO}_{2}$. These PET techniques confirmed the presence of penumbral tissue in human acute ischemic stroke ${ }^{5}$ and facilitated the generation of operational PET criteria for penumbra identification characterized by a preserved $\mathrm{CMRO}_{2}$, elevated OEF, and a reduced CBF. Data from $\mathrm{PET}^{6}$ also cemented the concept of tissue compartments after stroke, with small volumes of core and large volumes of penumbra being the favored pattern for reperfusion. These concepts have influenced clinical trial design ever since.

Recently, other PET ligands that aim to avoid some of the practical difficulties associated with ${ }^{15} \mathrm{O}$ multitracer PET have been investigated. These include radio-labeled flumazenil, which was reported to show decreased binding in irreversibly infarcted tissue, ${ }^{7}$ and radio-labeled fluoromisonidazole, which was reported to bind to viable but hypoxic tissue. ${ }^{8}$ However, recent rodent studies have challenged the specificity of binding to specific tissue compartments, and further investigation is required..$^{9,10}$

\section{What Do Current Routine Imaging Techniques Tell Us About Metabolism?}

NCCT simply provides data through the use of x-rays, with tissue attenuation decreasing in regions of cytotoxic edema. Parenchymal hypoattenuation has been associated with tissue destined for infarction on $\mathrm{PET}^{11}$ and a "core" pattern of perfusion on CT perfusion imaging. ${ }^{12}$ In addition, sulcal effacement in the absence of hypoattenuation has been shown to be associated with elevated $\mathrm{CBV}$, suggesting a "penumbral" perfusion pattern. ${ }^{12}$ However, it lacks sensitivity for early ischemic changes and for penumbra and cannot distinguish core from penumbra and from unaffected tissue in the hyperacute setting.

\section{MR Imaging}

DWI can detect subtle perturbations in the Brownian motion of water and is therefore more sensitive for cytotoxic edema than is CT. It is $>80 \%$ sensitive for ischemic stroke, ${ }^{13}$ and lesions are often considered to represent infarct core. However, back-to-back studies of MR imaging and PET show some voxels within the DWI lesion fulfill the PET criteria for penumbra. ${ }^{14}$ Observations of DWI reversal after reperfusion further challenged the assumed equivalence of a DWI lesion to core. ${ }^{15}$ However, recent data show that whereas there can be some reversal of very early lesions on DWI, such reversal is rarely complete ${ }^{16}$ and a lesion on DWI provides a reasonable, if not perfect, measure of infarct core in the acute setting. DWI does not, however, provide measures of $\mathrm{CMRO}_{2}$ but rather provides a marker of cytotoxic edema occurring as a result of reduced $\mathrm{CMRO}_{2}$. Therefore it cannot, by itself, define features of the penumbra such as hypoperfusion or OEF changes.

FLAIR sequences are heavily T2-weighted sequences with nulling of signal from CSF. FLAIR sequences detect vasogenic edema associated with established infarction. Because they are not sensitive to early cytotoxic edema, these sequences are inferior to DWI for the detection of acute stroke lesions and do not define core in the first hours after stroke onset. Pilot studies suggest that stroke lesions that carry a DWI-positiveFLAIR-negative signature are highly specific for the prediction of being $<3$ hours since onset, ${ }^{17}$ and the WAKE UP ${ }^{18}$ and MR WITNESS (www.clinicaltrials.gov) trials are underway to evaluate whether this imaging pattern may predict response to treatment in wake-up strokes. However, this imaging pattern aims to provide a surrogate marker of time rather than penumbra, and therefore FLAIR cannot provide direct measures of metabolic activity in the hyperacute setting.

A detailed critique of the use of PWI in acute ischemic stroke is beyond the scope of this article. Nonetheless, a number of key points should be noted. PWI data describe a dynamic signal intensity reflecting the perfusion of the tissue, including measures of CBF and CBV. Most studies have investigated MR-based PWI and have focused on regions with a larger PWI deficit compared with DWI deficit as being potentially salvageable - the PWI-DWI mismatch. ${ }^{19}$ Initial studies used a variety of methodologies ${ }^{20}$ and definitions ${ }^{21}$ for this mismatch, but the field is rapidly converging to agreement on the optimal imaging parameters that define this mismatch. ${ }^{22}$ It should be noted that CT perfusion may also be used to define tissue compartments using measures of hypoperfusion to define both penumbra and core. ${ }^{23}$ Although the explicit aim of the use of such patterns is to identify imaging signatures of favorable response to reperfusion therapy, the implication is that 


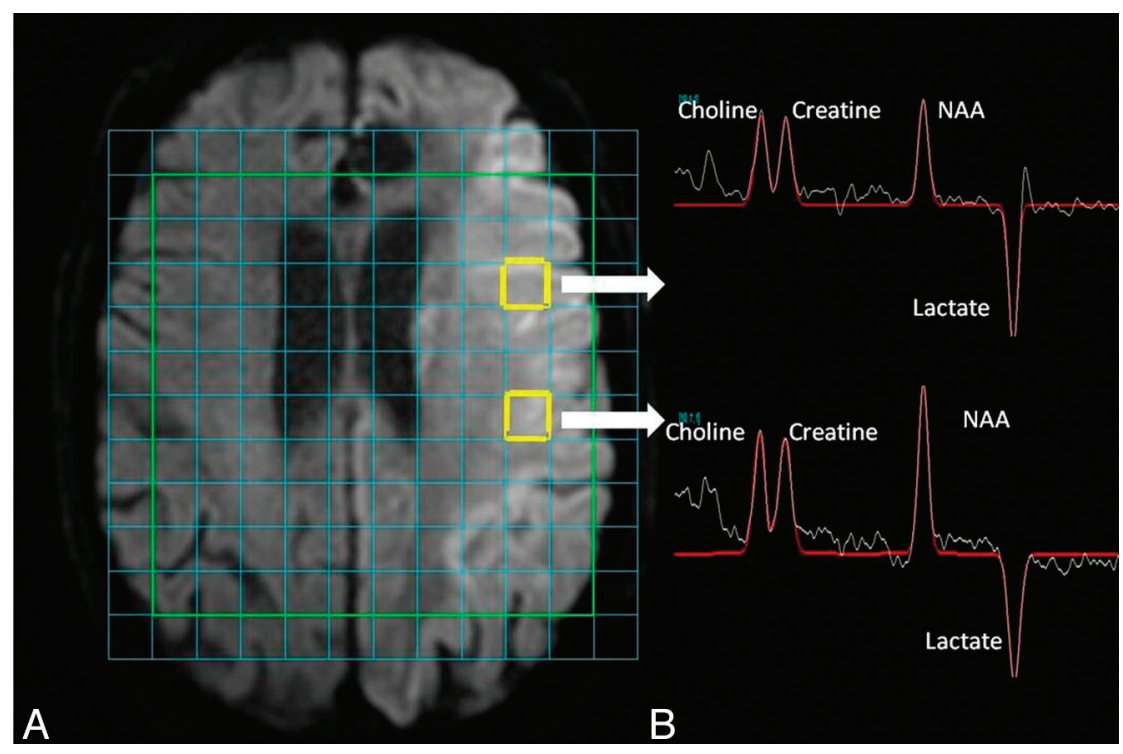

FIG 2. Data from MRS. A, Acute stroke lesion of the left hemisphere on DWI. There is a multivoxel spectroscopy grid overlaid onto the time. Users can select data from any voxel. The 2 voxels highlighted in yellow show the regions from which spectroscopy data were derived and are illustrated in on the right-hand side. B. Spectroscopy data for the 4 major metabolites that are annotated. NAA appears low, and lactate concentration is elevated. Area under the peaks indicates relative metabolite concentration. Although the lesion on DWI appears relatively uniform throughout, the NAA concentration is less in the anteriorly placed voxel compared with the posteriorly placed voxel.

patterns that predict a good response to reperfusion therapy have small volumes of core and large volumes of penumbra. Therefore, although the use of perfusion based measures does not define metabolic activity, they very clearly aim to identify different metabolic compartments. However, even though ongoing clinical trials may prove the clinical utility of such techniques, there will still be unanswered questions. For example, should all strictly defined PWI-DWI mismatch regions be considered equally or could metabolic imaging techniques further refine patient selection for treatment? For those with a matched PWI-DWI deficit, which (if any) patients will respond to reperfusion therapies within the time window?

The following sections discuss imaging techniques that may answer some of these questions.

\section{What Are the Emerging Metabolic Imaging Research Techniques?}

Proton MR Spectroscopy. MRS can be performed for in vivo human brain studies to measure metabolite concentrations derived from prespecified brain regions. Several nuclei can be studied, but because of their high concentration, ${ }^{1} \mathrm{H}$ nuclei (proton MRS) have been the focus. There are potentially a large number of metabolites that may be studied. However, for practical reasons, most studies have evaluated the concentrations of 4 main metabolites. NAA may be considered to be a marker of neuronal integrity, total creatine a marker of energy metabolism, choline a cell membrane marker, and lactate a marker of anaerobic glycolysis. The time course of metabolite concentration after stroke has been well characterized. ${ }^{24,25}$ After acute ischemic stroke, NAA falls within the first hour after arterial occlusion and continued decline is seen for up to 1 week after ictus. Creatine levels also fall after stroke, but changes in choline concentrations are more variable. Lactate concentration rises within minutes after stroke, and concentrations gradually decline after reperfusion.

One of the key questions of this technique is, can it provide useful data compared with current MR techniques? There are signals in the literature that at least some of the metabolite concentrations may already be reflected by the routine stroke protocol MR. NAA concentrations in "possibly abnormal" voxels on DWI are higher than those in "definitely abnormal" voxels. ${ }^{26}$ Higher concentrations of lactate are seen in the DWI lesion compared with the PWI-DWI mismatch region. ${ }^{27}$ Indeed, up to $41 \%$ of the variance of the metabolite concentration was predicted by the routine stroke protocol MR. ${ }^{28}$ However, although some of the remainder of the unaccounted variance may have been caused by "noise," it is likely that current MR protocols cannot provide us with as full a picture of metabolic activity as does MR spectroscopy. Indeed, we reported a case in which MRS demonstrated preserved NAA concentration with elevated lactate concentration in a DWI-negative patient imaged early after stroke, in a region that later further partially infarcted. ${ }^{28}$ However, the sensitivity of the identification of the "preserved NAA and elevated lactate" profile for the identification of penumbral tissue was questioned in that study. If lactate sensitivity can be optimized, however, identification of raised lactate that reverses with transient hyperoxia may be another route by which potentially salvageable tissue may be identified. ${ }^{27,29}$ MRS may also be able to show differences in neuronal integrity across a DWI lesion (Fig 2).

A number of limitations have been identified with the use of a clinically applicable MRS sequence in acute ischemic stroke. The technique has a low SNR, and shortening the sequence duration in acute stroke further compounds these problems. Moreover, movement of patients with acute stroke will increase the chances of lipid contamination of signal.

Despite these limitations, MRS may still find a niche, and this may include its use in clinical trials. For example, our recent description of the detection of elevated levels of reduced glutathione in a small number of patients with subacute stroke suggests that a repeatable in vivo marker of oxidative stress in stroke is now available. $^{30}$ This could be used to confirm a tissue effect from investigational medicinal products in clinical trials as a surrogate marker of outcome.

${ }^{17} \mathrm{O}$ Imaging. Although most MRS studies have exploited proton nuclei, some studies have focused on oxygen nuclei. Unlike the more abundant ${ }^{16} \mathrm{O},{ }^{17} \mathrm{O}$ has MR visibility. Although it is naturally occurring, it is present in very low concentrations in human tissue. ${ }^{17} \mathrm{O}$ may be detected directly by use of MRS or indirectly by detecting ${ }^{17} \mathrm{O}$-labeled water by exploiting the principle of cou- 
pling between protons and ${ }^{17} \mathrm{O}$ by use of spin-echo MR images, both after ${ }^{17} \mathrm{O}$ inhaled gas. The MR visibility makes assessment of results fairly straightforward because ${ }^{17} \mathrm{O}$ is only detectable when oxidative metabolism is complete and $\mathrm{H}_{2}{ }^{17} \mathrm{O}$ is produced. Therefore, confounds by signal from oxygen at other stages in the metabolic pathway are avoided. $\mathrm{CMRO}_{2}$ may be calculated if the perfusion-related clearance rate of water and recirculation characteristics are considered. In addition, Zhu et $\mathrm{al}^{31}$ have recently shown that the technique may also be used to determine $\mathrm{CBF}$. The direction of changes in $\mathrm{CMRO}_{2}, \mathrm{CBF}$, and OEF reported from $\mathrm{PET}$ studies after stroke has also been described in a recent murine study by use of ${ }^{17} \mathrm{O}$ imaging. ${ }^{32}$ Human imaging by use of ${ }^{17} \mathrm{O}$ has been shown to be safe ${ }^{33}$ and the production of $\mathrm{CMRO}_{2}$ maps to be feasible in human volunteers. ${ }^{34}$ Remaining limitations for the translation of this technique into humans include the different kinetics of ${ }^{17} \mathrm{O}$ in humans compared with small animals and the requirement for high field strengths to optimize signal. Therefore, human studies remain limited.

Sodium Imaging. It is well established that as adenosine triphosphate generation becomes insufficient to maintain cellular sodium-potassium adenosine triphosphatase pumps after ischemic stroke, there is influx of sodium into cells. Imaging tissue sodium therefore provides an attractive potential surrogate marker of sodium-potassium adenosine triphosphatase pump dysfunction that occurs in infarct core. Although the SNR of sodium imaging techniques is low, the production of image maps of sodium intensity is now feasible. A number of animal studies have confirmed an increase in brain tissue sodium concentration occurring after stroke ${ }^{35-37}$ by use of field strengths of between $3 \mathrm{~T}$ and 9.4T. Hussain et $\mathrm{al}^{38}$ imaged 21 patients with stroke and demonstrated that in the first 7 hours, there was only a very modest increase in tissue sodium $(<10 \%)$, but this increased more rapidly after 7 hours and plateaued at 48 hours. The authors postulated that sodium imaging may be used as a tissue clock, with potential utility for evaluation of wake-up strokes. A further study confirmed that there were no sodium changes in the PWI-DWI mismatch region. ${ }^{39}$ Moreover, unlike the ADC derived from DWI, which was statically low over the first few days of infarction, sodium intensity steadily increased through this time. This suggests that sodium imaging may provide additional information in comparison to DWI, even though both are measures of cytotoxic edema. The study authors postulated that a potential niche for sodium may be in patient selection for reperfusion therapies, whereby the optimal imaging profile might be a PWI-DWI mismatch without any significant changes seen on sodium imaging.

Although it is an intriguing proposition, there are a number of potentially significant barriers to the widespread adoption of this technique. First, although feasible on 3T machines, higher field strengths would be advantageous, and these are not clinically routine at present. Second, low SNR also necessitates long imaging times. Third, it is still far from clear the additional clinical utility sodium imaging would provide. Studies of DWI-FLAIR mismatch to provide a "tissue clock" are well underway, and these imaging techniques are already part of the stroke MR protocol. Perhaps sodium imaging may further refine the definition of core and distinguish regions of DWI lesions that are penumbral and are already core.
T2*-Weighted MR Imaging. The ability of PET to measure both $\mathrm{CMRO}_{2}$ and OEF simultaneously, in addition to providing measures of $\mathrm{CBF}$ and $\mathrm{CBV}$, has ensured its status as the reference standard research metabolic imaging technique. Recently, attention has been paid to MR images sensitive to deoxyhemoglobin, because its concentration in the cerebral venous circulation changes as OEF varies in the presence of maintained $\mathrm{CMRO}_{2}$. $\mathrm{T} 2{ }^{\star} \mathrm{WI}$ sequences are sensitive to magnetic susceptibility differences between blood and tissue parenchyma created by paramagnetic substances present in the tissue microcirculation, of which deoxyhemoglobin is one. As OEF and therefore venous deoxyhemoglobin increases in the penumbra, one would expect parenchyma and venous structures to appear dark. Indeed, Morita et $\mathrm{al}^{40}$ reported such findings on $\mathrm{T} 2^{\star} \mathrm{WI}$ gradient-echo sequences, which are routinely used to detect intracerebral hemorrhage. Similar findings have been reported on precontrast $\mathrm{T} 2 *$-weighted PWI sequences in human and rodent studies. ${ }^{41,42}$ However, in a study that measured T2' (T2* corrected for spin-spin effects), although differences in signal between core, penumbra, and healthy tissue were noted, wide confidence intervals precluded the determination of discriminative thresholds. ${ }^{43}$ Indeed, back-toback PET and MR studies failed to confirm a correlation between $\mathrm{T} 2{ }^{\star} \mathrm{WI}$ signal intensity on precontrast $\mathrm{T} 2{ }^{\star}$-weighted PWI and OEF measures derived from PET imaging. ${ }^{44}$ This may be in part due to the fact that $\mathrm{T} 2^{\star} \mathrm{WI}$ signal is influenced by a number of factors including the increase in $\mathrm{T} 2{ }^{\star} \mathrm{WI}$ signal occurring a number of hours after onset of stroke caused by vasogenic edema.

Owing to the limitations of static measures of T2WI or T2*WI signal intensity to provide measures of metabolic activity, other MR imaging measures have been investigated, including oxygen challenge imaging (OCI) and also potentially quantitative measures of $\mathrm{CMRO}_{2}$.

Oxygen Challenge Imaging. OCI uses transient hyperoxia during $\mathrm{T} 2{ }^{*}$-weighted MR images to demonstrate dynamic changes in deoxyhemoglobin concentration. The hypothesis of the technique is that in healthy actively metabolizing tissue in which the OEF is approximately $0.3-0.4$, supplementary oxygen will aid the conversion of cerebral venous deoxyhemoglobin to oxyhemoglobin, which will be associated with a $\mathrm{T} 2^{\star} \mathrm{WI}$ signal intensity increase. In the penumbra, in which one would expect an increase in $\mathrm{CBV}, \mathrm{OEF}$, and therefore deoxyhemoglobin, an exaggerated increase in $\mathrm{T} 2{ }^{\star} \mathrm{WI}$ signal intensity would be expected. In contrast, in infarct core, one would expect a diminished or absent response to OCI. Santosh et $\mathrm{al}^{45}$ confirmed these results in a rat model of MCA occlusion. With the use of $\left[{ }^{14} \mathrm{C}\right] 2$-deoxyglucose autoradiography, the group subsequently showed preserved metabolic activity in the $\mathrm{T} 2^{\star} \mathrm{WI}$ signal-defined penumbra, and the exaggerated $\mathrm{T} 2{ }^{*} \mathrm{WI}$ signal was seen to normalize after reperfusion, consistent with the underlying hypothesis of the technique. ${ }^{46,47}$ Rodent studies by another group ${ }^{48}$ showed that the region of exaggerated OCI response was larger than the PWI-DWI mismatch region, suggesting that the technique may also be able to detect potentially salvageable tissue within the DWI lesion. A pilot human study was broadly consistent with the results from animal studies ${ }^{49}$ with diminished OCI response in DWI lesions (Fig 3). Unfortunately, small volumes of PWI-DWI mismatch in small numbers of subjects did not allow statistically significant 


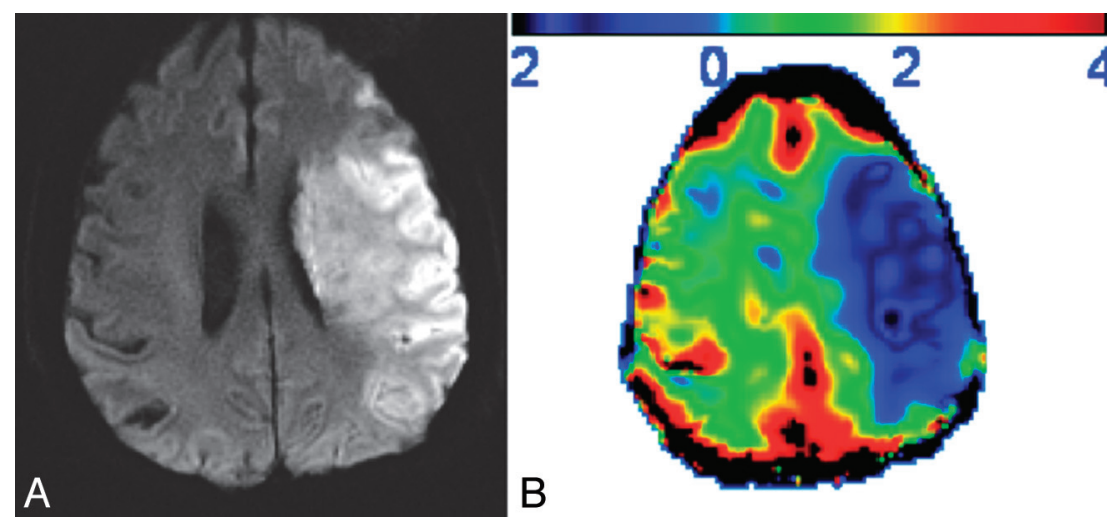

FIG 3. An example of oxygen challenge imaging. $A$, Stroke lesion of the left hemisphere on DWI from a subject last seen well approximately 24 hours before imaging. $B$, Results from oxygen challenge imaging. The percentage change in $\mathrm{T}^{*} \mathrm{WI}$ signal is indicated in the scale bar at the top (positive results indicate an increase in signal; negative results a decrease in signal). There is a diminished response to oxygen challenge in regions corresponding to the established lesion on DWI, suggesting a core pattern.

proof of exaggerated OCI penumbral response, though some encouraging patterns were described.

Although studies of OCI are still at an early stage, further validation studies are required to answer remaining questions. For example, are different oxygen concentrations required to image different tissue compartments? Does impaired vascular autoregulation affect results in humans? Which thresholds distinguish tissue compartments?

MR Measures of $\mathrm{CMRO}_{2}$. Whereas some groups have investigated $\mathrm{T} 2{ }^{\star} \mathrm{WI}$ sequences by use of the oxygen challenge technique, others have modeled either the T2WI or T2*WI signal to calculate metabolic parameters. By exploiting the "static dephasing regime" of Yablonskiy and Haake, ${ }^{50}$ which states that above a particular echo time a component of the T2*WI signal becomes dependent on OEF, the magnetic susceptibility of blood, venous CBV, hematocrit, and static magnetic field, An and $\operatorname{Lin}^{51}$ used a multiecho gradient and spin-echo sequence to provide measures of cerebral venous oxygen saturation and OEF. This measure can be combined with CBF measures to calculate the MR equivalent of $\mathrm{CMRO}_{2}$ - the cerebral metabolic oxygen index (MR COMI). The group has since validated their findings in animals and healthy volunteers, and in general results have been consistent with the PET literature. The first stroke study was completed a decade ago, ${ }^{52}$ and in 7 patients imaged after an average of 7.5 hours post ictus, the putative core showed a diminished MR imaging-COMI value, as expected. The group has recently described 41 patients with stroke with baseline imaging at 3 hours post ictus and defined MR-COMI thresholds to predict the inner and outer border of the ischemic penumbra, ${ }^{53}$ with encouraging results. Although optimistic, the authors concluded that a large multicenter study is required to determine the clinical utility of these sequences.

pH Imaging. Significant hypoperfusion precipitates a switch from aerobic to anaerobic metabolism with subsequent lactate production. One of the limitations of MRS for the detection of lactate is the limited spatial resolution, even with multivoxel MRS. Therefore, in an effort to identify the ischemic penumbra, other modal- ities to identify the tissue that has switched to anaerobic glycolysis have been investigated. Chemical exchange processes between amide protons of cellular peptides and proteins occurs in a $\mathrm{pH}$-dependent fashion, and methods for detecting this chemical exchange have already been developed $^{54}$ : chemical exchange saturation transfer. Zhou et al ${ }^{55}$ coined the term amide proton transfer to describe the chemical exchange saturation transfer effect between water and protons. Given that amide proton transfer is $\mathrm{pH}$ dependent, measures of amide proton transfer may aid the measurement of $\mathrm{pH}$. Most studies investigating this have been performed in rodents. Initial studies demonstrated the feasibility of detecting a reduced $\mathrm{pH}$ in the ischemic hemisphere, ${ }^{55}$ and subsequent studies have illustrated a number of features of lesions on $\mathrm{pH}$-weighted imaging that are consistent with penumbra. First, a strong correlation between tissue $\mathrm{pH}$ and lactate concentration has been observed. ${ }^{56}$ Next, the abnormalities precede lesions on $\mathrm{ADC}$ and are bigger or equal to the volume of ADC lesions but smaller or equal to the volume of perfusion deficits in all rats. Finally, regions with reduced $\mathrm{pH}$ proceeded to infarction. ${ }^{57}$ These data suggest that the use of $\mathrm{pH}$ imaging may help to define the ischemic penumbra whereby hypoperfused tissue with normal $\mathrm{ADC}$ and $\mathrm{pH}$ represents benign oligemia, and hypoperfused tissue with normal $\mathrm{ADC}$ and low $\mathrm{pH}$ may represent ischemic penumbra. A further study evaluated multimodal MR, including $\mathrm{pH}$ imaging, in a rodent model of stroke to predict tissue outcome. ${ }^{58}$ The authors concluded that the addition of $\mathrm{pH}$-weighted imaging data to a model to predict tissue outcome was superior to the use of PWIDWI data alone.

The use of $\mathrm{pH}$-weighted imaging in human data has so far been scarce, largely slowed by technical complexities in the translation of this technique to humans. However, study of a small number of volunteers and patients with stroke has confirmed the feasibility of this technique at $3 \mathrm{~T}$, in addition to the ability to detect low tissue $\mathrm{pH}$ in the stroke region. ${ }^{59}$

\section{CONCLUSIONS}

Imaging techniques included in current multimodal CT and MR protocols provide a wealth of information, including providing surrogate markers of metabolic activity. An immediate goal is to gain better understanding of how to use these techniques to make therapeutic decisions — several studies are ongoing in this regard. There are many techniques that are at an earlier stage of investigation for imaging penumbra and core (Figs 4 and 5); we have discussed some but not all of these here and have not discussed techniques which aim to image other processes such as inflammation. While promising, the emerging metabolic imaging techniques require substantial validation to determine how they would fit into current imaging protocols and precisely what information can be added that cannot already be inferred from existing techniques. Many of these will require technical improvements before being feasible for use in hyperacute stroke. Despite 


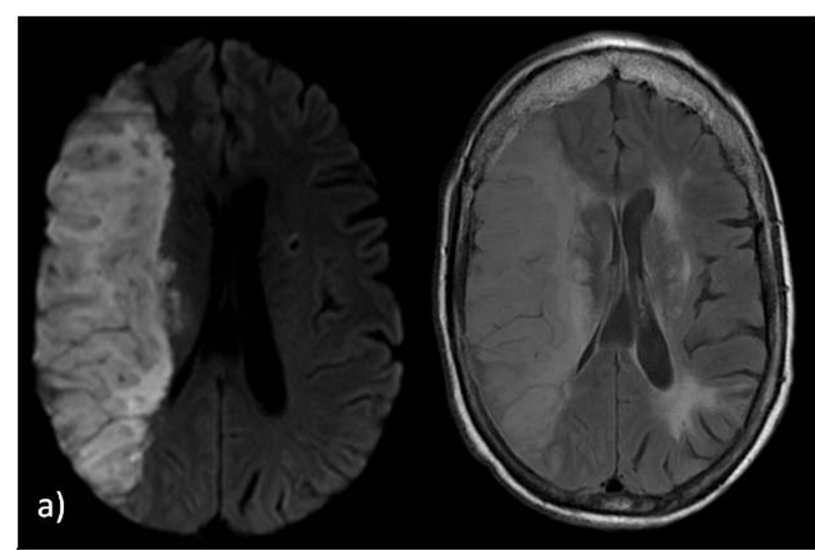

b) Potential Novel Markers of Core

- MRS - IOW NAA

-MRS -no reversal of lactate with hyperoxia

- $\mathrm{OCl}$ - diminished $\mathrm{T} 2{ }^{*}$-weighted signal change

- $\mathrm{pH}$ imaging - low $\mathrm{pH}$, low CBF, low ADC

- MR-COMI - - low $\mathrm{CMRO}_{2}$

.17 O imaging - low $\mathrm{CMRO}_{2}$

FIG 4. Current and potential markers of core. $A, M R$ images of DWI (right) and FLAIR image (left) showing extensive established infarct core; $B$, list of potential imaging profiles that may refine definitions of core. OCl indicates oxygen challenge imaging; MR-COMI, MR cerebral oxygen metabolic index; $\mathrm{CMRO}_{2}$, cerebral metabolic rate for oxygen.

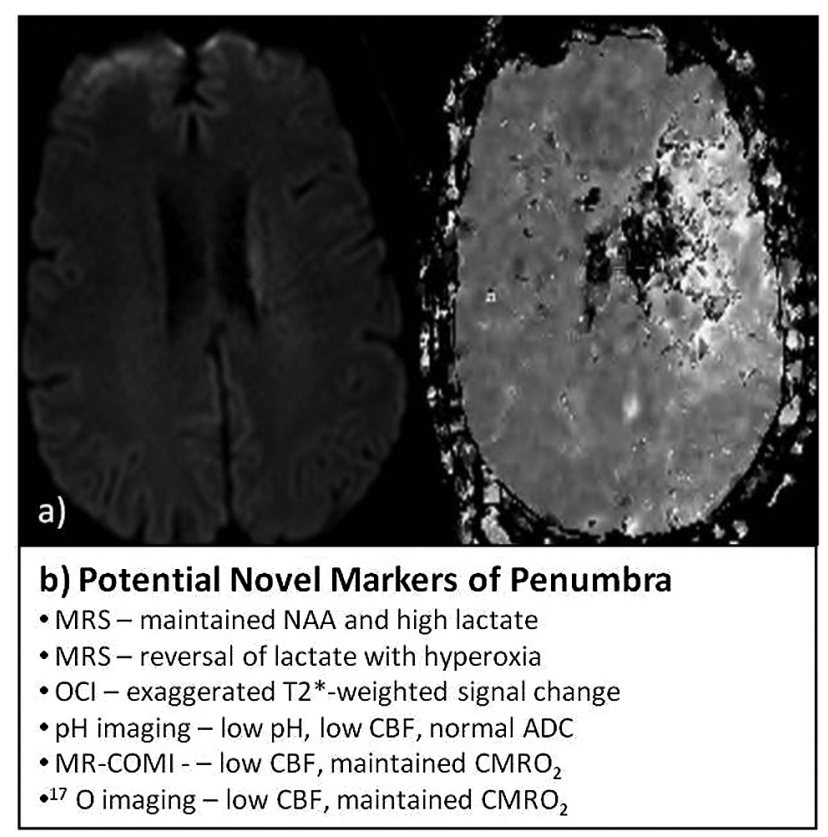

FIG 5. Current and potential markers of penumbra. $A$, MR images of DWI (right) and PWI (left) showing a region of PWI-DWI mismatch; $B$, list of potential imaging profiles that may refine definitions of penumbra. $\mathrm{OCl}$ indicates oxygen challenge imaging; MR-COMI, MR cerebral oxygen metabolic index; $\mathrm{CMRO}_{2}$, cerebral metabolic rate for oxygen.

many uncertainties surrounding these novel techniques, there is one thing for certain: we will see plenty more studies over the next few years.

Disclosures: Krishna Dani-UNRELATED: Grants/Grants Pending: Chief Scientists Office Scotland, ${ }^{*}$ Neurosciences Foundation Glasgow, ${ }^{*}$ Stroke Association,* National Health Service UK (NHS)* endowment funds, Comments: Numerous unrelated grants in the field of neuroimaging; Payment for Development of Educational Presentations: Later Life Learning, Comments: Basics of Stroke; OTHER RELATIONSHIPS: I am currently involved in studies of metabolic imaging.

\section{REFERENCES}

1. Astrup J, Siesjo BK, Symon L. Thresholds in cerebral ischemia: the ischemic penumbra. Stroke 1981;12:723-5

2. Furlan M, Marchal G, Viader F, et al. Spontaneous neurological recovery after stroke and the fate of the ischemic penumbra. Ann Neurol 1996;40:216-26

3. National Institute of Neurological Disorders and Stroke rt-PA Stroke Study Group. Tissue plasminogen activator for acute ischemic stroke. N Engl J Med 1995;333:1581-7

4. Heiss WD, Huber M, Fink GR, et al. Progressive derangement of periinfarct viable tissue in ischemic stroke. J Cereb Blood Flow Metab 1992;12:193-203

5. Marchal G, Beaudouin V, Rioux P, et al. Prolonged persistence of substantial volumes of potentially viable brain tissue after stroke: a correlative PET-CT study with voxel-based data analysis. Stroke 1996;27:599-606

6. Marchal G, Serrati C, Rioux P, et al. PET imaging of cerebral perfusion and oxygen consumption in acute ischaemic stroke: relation to outcome. Lancet 1993;341:925-7

7. Heiss WD, Kracht L, Grond M, et al. Early [(11)C]Flumazenil/H(2)O positron emission tomography predicts irreversible ischemic cortical damage in stroke patients receiving acute thrombolytic therapy. Stroke 2000;31:366-9

8. Read SJ, Hirano T, Abbott DF, et al. Identifying hypoxic tissue after acute ischemic stroke using PET and 18F-fluoromisonidazole. $\mathrm{Neu}$ rology 1998;51:1617-21

9. Rojas S, Martin A, Pareto D, et al. Positron emission tomography with C-11-flumazenil in the rat shows preservation of binding sites during the acute phase after $2 \mathrm{~h}$-transient focal ischemia. $\mathrm{Neu}$ roscience 2011;182:208-16

10. Spratt NJ, Donnan GA, McLeod DD, et al. 'Salvaged' stroke ischaemic penumbra shows significant injury: studies with the hypoxia tracer FMISO. J Cereb Blood Flow Metab 2011;31:934-43

11. Grond M, Von Kummer R, Sobesky J, et al. Early x-ray hypoattenuation of brain parenchyma indicates extended critical hypoperfusion in acute stroke. Stroke 2000;31:133-9

12. Muir KW, Baird-Gunning J, Walker L, et al. Can the ischemic penumbra be identified on noncontrast CT of acute stroke? Stroke 2007;38:2485-90

13. Chalela JA, Kidwell CS, Nentwich LM, et al. Magnetic resonance imaging and computed tomography in emergency assessment of patients with suspected acute stroke: a prospective comparison. Lancet 2007;369:293-98

14. Guadagno JV, Warburton EA, Jones PS, et al. How affected is oxygen metabolism in DWI lesions? A combined acute stroke PET-MR study. Neurology 2006;67:824-9

15. Kidwell CS, Saver JL, Mattiello J, et al. Thrombolytic reversal of acute human cerebral ischemic injury shown by diffusion/perfusion magnetic resonance imaging. Ann Neurol 2000;47:462-9

16. Freeman JW, Luby M, Merino JG, et al. Negative diffusion-weighted imaging after intravenous tissue-type plasminogen activator is rare and unlikely to indicate averted infarction. Stroke 2013;44:1629-34

17. Thomalla G, Rossbach P, Rosenkranz M, et al. Negative fluid-attenuated inversion recovery imaging identifies acute ischemic stroke at 3 hours or less. Ann Neurol 2009;65:724-32

18. Thomalla G, Ebinger M, Fiehler J, et al. EU-funded treatment study: WAKE-UP: a randomized, placebo-controlled MRI-based trial of thrombolysis in wake-up stroke. Nervenarzt 2012;83:1241-51

19. Schlaug G, Benfield A, Baird AE, et al. The ischemic penumbra: operationally defined by diffusion and perfusion MRI. Neurology 1999;53:1528-37

20. Dani KA, Thomas RGR, Chappell FM, et al. Systematic review of perfusion imaging with computed tomography and magnetic resonance in acute ischemic stroke: heterogeneity of acquisition and postprocessing 
parameters a translational medicine research collaboration multicentre acute stroke imaging study. Stroke 2012;43:563-66

21. Dani KA, Thomas RGR, Chappell FM, et al. Computed tomography and magnetic resonance perfusion imaging in ischemic stroke: definitions and thresholds. Ann Neurol 2011;70:384-401

22. Olivot JM, Mlynash M, Thijs VN, et al. Optimal Tmax threshold for predicting penumbral tissue in acute stroke. Stroke 2009;40:469-75

23. Wintermark M, Flanders AE, Velthuis B, et al. Perfusion-CT assessment of infarct core and penumbra: receiver operating characteristic curve analysis in $\mathbf{1 3 0}$ patients suspected of acute hemispheric stroke. Stroke 2006;37:979-85

24. Gideon P, Henriksen O, Sperling B, et al. Early time course of Nacetylaspartate, creatine and phosphocreatine, and compounds containing choline in the brain after acute stroke: a proton magnetic resonance spectroscopy study. Stroke 1992;23:1566-72

25. Saunders DE, Howe FA, van den Boogaart A, et al. Continuing ischemic damage after acute middle cerebral artery infarction in humans demonstrated by short-echo proton spectroscopy. Stroke 1995;26:1007-13

26. Munoz Maniega S, Cvoro V, Chappell FM, et al. Changes in NAA and lactate following ischemic stroke: a serial MR spectroscopic imaging study. Neurology 2008;71:1993-99

27. Singhal AB, Ratai E, Benner T, et al. Magnetic resonance spectroscopy study of oxygen therapy in ischemic stroke. Stroke 2007;38:2851-4

28. Dani KA, An L, Henning EC, et al. Multivoxel MR spectroscopy in acute ischemic stroke comparison to the stroke protocol MRI. Stroke 2012;43:2962-67

29. Holmes WM, Lopez-Gonzalez MR, Gallagher L, et al. Novel MRI detection of the ischemic penumbra: direct assessment of metabolic integrity. NMR Biomed 2012;25:295-304

30. An L, Dani KA, Shen J, et al. Pilot results of in vivo brain glutathione measurements in stroke patients. J Cereb Blood Flow Metab 2012;32:2118-21

31. Zhu $\mathrm{X}$, Zhang $\mathrm{Y}$, Wiesner $\mathrm{H}$, et al. In vivo measurement of CBF using (17) O NMR signal of metabolically produced $\mathrm{H}(2)$ (17) $\mathrm{O}$ as a perfusion tracer. Magn Reson Med 2013;70:309-14

32. Zhu X-H, Chen JM, Tu T-W, et al. Simultaneous and noninvasive imaging of cerebral oxygen metabolic rate, blood flow and oxygen extraction fraction in stroke mice. Neuroimage 2013;64:437-47

33. Atkinson IC, Sonstegaard R, Pliskin NH, et al. Vital signs and cognitive function are not affected by 23 -sodium and 17-oxygen magnetic resonance imaging of the human brain at 9.4 T. J Magn Reson Imaging 2010;32:82-87

34. Atkinson IC, Thulborn KR. Feasibility of mapping the tissue mass corrected bioscale of cerebral metabolic rate of oxygen consumption using 17-oxygen and 23-sodium MR imaging in a human brain at 9.4 T. Neuroimage 2010;51:723-33

35. Thulborn KR, Gindin TS, Davis D, et al. Comprehensive MR imaging protocol for stroke management: tissue sodium concentration as a measure of tissue viability in nonhuman primate studies and in clinical studies. Radiology 1999;213:156-66

36. Wetterling F, Ansar S, Handwerker E. Sodium-23 magnetic resonance imaging during and after transient cerebral ischemia: multinuclear stroke protocols for double-tuned $\mathrm{Na}-23 / \mathrm{H}-1$ resonator systems. Physics Med Biol 2012;57:6929-46

37. Heiler PM, Langhauser FL, Wetterling F, et al. Chemical shift sodium imaging in a mouse model of thromboembolic stroke at 9.4 T. J Magn Reson Imaging 2011;34:935-40

38. Hussain MS, Stobbe RW, Bhagat YA, et al. Sodium imaging intensity increases with time after human ischemic stroke. Ann Neurol 2009;66:55-62

39. Tsang A, Stobbe RW, Asdaghi N, et al. Relationship between sodium intensity and perfusion deficits in acute ischemic stroke. J Magn Reson Imaging 2011;33:41-47

40. Morita N, Harada M, Uno $\mathrm{M}$, et al. Ischemic findings of $\mathbf{T} 2{ }^{\star}$ weighted 3-Tesla MRI in acute stroke patients. Cerebrovasc Dis 2008;26:367-75

41. Tamura H, Hatazawa J, Toyoshima H, et al. Detection of deoxygenation-related signal change in acute ischemic stroke patients by T2*-weighted magnetic resonance imaging. Stroke 2002;33:967-71

42. Wardlaw JM, von Heijne A. Increased oxygen extraction demonstrated on gradient echo $\left(\mathrm{T} 2^{\star}\right)$ imaging in a patient with acute ischaemic stroke. Cerebrovasc Dis 2006;22:456-58

43. Geisler BS, Brandhoff F, Fiehler J, et al. Blood-oxygen-level-dependent MRI allows metabolic description of tissue at risk in acute stroke patients. Stroke 2006;37:1778-84

44. Donswijk ML, Jones PS, Guadagno JV, et al. T2*-weighted MRI versus oxygen extraction fraction PET in acute stroke. Cerebrovasc Dis 2009;28:306-13

45. Santosh C, Brennan D, McCabe C, et al. Potential use of oxygen as a metabolic biosensor in combination with $\mathrm{T} 2{ }^{*}$-weighted MRI to define the ischemic penumbra. J Cereb Blood Flow Metab 2008;28:1742-53

46. Robertson CA, McCabe C, Gallagher L, et al. Stroke penumbra defined by an MRI-based oxygen challenge technique, 1: validation using [(14)C]2-deoxyglucose autoradiography. J Cereb Blood Flow Metab 2011;31:1778-87

47. Robertson CA, McCabe C, Gallagher L, et al. Stroke penumbra defined by an MRI-based oxygen challenge technique, 2: validation based on the consequences of reperfusion. J Cereb Blood Flow Metab 2011;31:1788-98

48. Shen Q, Huang S, Du F, et al. Probing ischemic tissue fate with BOLD fMRI of brief oxygen challenge. Brain Res 2011;1425:132-41

49. Dani KA, Santosh C, Brennan D, et al. T2*-weighted magnetic resonance imaging with hyperoxia in acute ischemic stroke. Ann Neurol 2010;68:37-47

50. Yablonskiy DA, Haacke EM. Theory of NMR signal behavior in magnetically inhomogeneous tissues: the static dephasing regime. Magn Reson Med 1994;32:749-63

51. An H, Lin W. Quantitative measurements of cerebral blood oxygen saturation using magnetic resonance imaging. J Cereb Blood Flow Metab 2000;20:1225-36

52. Lee JM, Vo KD, An H, et al. Magnetic resonance cerebral metabolic rate of oxygen utilization in hyperacute stroke patients. Ann Neurol 2003;53:227-32

53. Lin W, An H, Ford AL, et al. MR imaging of oxygen extraction and neurovascular coupling. Stroke 2013;44:S61-64

54. Ward KM, Aletras AH, Balaban RS. A new class of contrast agents for MRI based on proton chemical exchange dependent saturation transfer (CEST). J Magn Reson 2000;143:79-87

55. Zhou JY, Payen JF, Wilson DA, et al. Using the amide proton signals of intracellular proteins and peptides to detect $\mathrm{pH}$ effects in MRI. Nat Med 2003;9:1085-90

56. Sun PZ, Cheung JS, Wang E, et al. Association between $\mathbf{p H}$-weighted endogenous amide proton chemical exchange saturation transfer MRI and tissue lactic acidosis during acute ischemic stroke. J Cereb Blood Flow Metab 2011;31:1743-50

57. Sun PZ, Zhou J, Sun W, et al. Detection of the ischemic penumbra using pH-weighted MRI. J Cereb Blood Flow Metab 2007;27:1129-36

58. Jokivarsi KT, Hiltunen Y, Tuunanen PI, et al. Correlating tissue outcome with quantitative multiparametric MRI of acute cerebral ischemia in rats. J Cereb Blood Flow Metab 2010;30:415-27

59. Zhao X, Wen Z, Huang F, et al. Saturation power dependence of amide proton transfer image contrasts in human brain tumors and strokes at 3 T. Magn Reson Med 2011;66:1033-41 